\title{
Effect of primary decompressive craniectomy on outcomes in severe traumatic brain injury with mass lesions and the independent predictors for operation decision
}

Chen Yang

Xi'an Tangdu Hospital of No4 Military Medical University

Jia-Rui Zhang

Xi'an Tangdu Hospital of No4 Military Medical University

Gang Zhu

Xi'an Tangdu Hospital of No4 Military Medical University Hao Guo

Xi'an Tangdu Hospital of No4 Military Medical University

Fei Gao

Xi'an Tangdu Hospital of No4 Military Medical University

Bao Wang

Xi'an Tangdu Hospital of No4 Military Medical University

Wen-Xing Cui

Xi'an Tangdu Hospital of No4 Military Medical University

Ying-Wu Shi

Xi'an Tangdu Hospital of No4 Military Medical University

Yong Du

Xi'an Tangdu Hospital of No4 Military Medical University

Zhi-Hong Li

Xi'an Tangdu Hospital of No4 Military Medical University

Liang Wang

Xi'an Tangdu Hospital of No4 Military Medical University

Lian-Ting Ma

PLA Army General Hospital

Yan Qu ( $\square$ yanqu0123@fmmu.edu.cn )

Xi'an Tangdu Hospital of No4 Military Medical University

Shunnan Ge ( $\boldsymbol{D}$ gsn8561@hotmail.com )

Xi'an Tangdu Hospital of No4 Military Medical University

https://orcid.org/0000-0002-3881-3950 
Research

Keywords: Basal cisterns, Craniotomy, Decompressive craniectomy, Pupillary light reflex, Traumatic brain injury

Posted Date: August 19th, 2020

DOI: https://doi.org/10.21203/rs.3.rs-58888/v1

License: (c) (i) This work is licensed under a Creative Commons Attribution 4.0 International License.

Read Full License 


\section{Abstract}

Background: Although operative indications for traumatic brain injury (TBI) have been evaluated, neurosurgeons often face a dilemma of whether or not to remove the bone flap after mass lesion evacuation, and a useful predictive scoring model for which patients should be decompressive craniectomy (DC) has yet to be developed. The aim of this study was firstly to compare the outcomes of craniotomy and $\mathrm{DC}$, and secondly to determine independent predictors and develop a multivariate logistic regression equation to determine whom should perform primary DC in TBI patients with mass lesions.

Methods: A total of nine different variables were evaluated. All 245 patients with severe TBI in this study were retrospectively evaluated between June 2015 and May 2019 and all underwent decompressive craniectomy (DC) or craniotomy for mass lesion removal. The 6-month mortality and Extended Glasgow Outcome Scale (GOSE) were compared between DC and craniotomy. By using univariate, multiple logistic regression and prognostic regression scoring equations it was possible to draw Receiver Operating Characteristic curves (ROC) to predict the decision for DC.

Results: The overall 6-month mortality in the entire cohort was $11.43 \%(28 / 245)$. DC patients had a lower mean preoperative Glasgow Coma Scale (GCS) ( $p=0.01)$; more patients with GCS of $6(p=0.007)$;more unresponsive pupillary light reflex ( $p<0.001)$; more closed basal cisterns $(p<0.001)$; and more patients with diffuse injury $(\mathrm{p}=0.025)$ than craniotomy patients. Given the greater severity, patients undergoing primary DC had higher 6-month mortality than the remainder of the cohort. However, in the surviving patients, the favorable GOSE rate was similar in two groups. We found that pupillary light reflex and basal cisterns were independent predictors for DC decision. Using ROC curve to predict the probability of DC, the sensitivity was $81.6 \%$ and the specificity was $84.9 \%$.

Conclusion: Our preliminary findings showed that the primary DC may benefit subgroups of STBI with mass lesions, and unresponsive pre-op pupil reaction, and closed basal cistern to predict the DC decision were useful. These sensitive variables can be used as a referential guideline in our daily practice to decide to perform or avoid primary DC.

\section{Introduction}

Traumatic brain injury (TBI) presents a great challenge to public health worldwide[1]. In China, TBI is a problem primarily of young and middle aged adults and the proportion of patients with severe TBI is as high as around $20 \%$, leading to huge losses in health and labor capacity[2, 3]. Although most small parenchymal lesions do not require surgical evacuation[4], the development of mass effect from larger lesions may result in secondary brain injury, placing the patient at risk of progressive neurological deterioration, refractory intracranial hypertension, herniation, and death[5]. Craniotomy with evacuation of the hematoma is the standard surgical treatment for these patients. A significant proportion of patients, especially severe TBI patients, however, have intractable intracranial hypertension after standard craniotomy, which necessitates craniectomy, and these patients have the worst outcome[6]. However, 
there is a paucity of evidence in the literature regarding the best surgical strategy (primary decompressive craniectomy or craniotomy) for this group of patients and surgical decision making is often haphazard[7].

Decompressive craniectomy (DC) is a surgical procedure in which a large component of the bony skull is removed and the underlying dura mater is opened[8].Primary DC refers to leaving a large bone flap out after the evacuation of an intracranial hematoma in the early phase after a TBI[9]. DC holds the promise of reduced mortality, but long term neurological outcomes and indications remain controversial[10]. However, the indications for DC remain difficult to define for the surgeon in the emergency setting[11]. The current debate centers on patient selection and clinical indications[12].Intracranial pressure monitoring is widely used to evaluate the intracranial status of patients with TBI[1]. In the two decades, the addition of intracranial pressure monitoring has markedly improved decision making and management of patients with severe TBI[13]. However, in China, most patients admitted to the intensive care unit (ICU) with severe TBI did not receive an intracranial pressure (ICP) monitoring device[2], reflect the role of clinical assessment and imaging in making treatment decisions, or economic constraints in case of non-reimbursement[14]. In contrast, computed tomography (CT) scans are done for patients with $\mathrm{TBI}$ in all hospitals with departments of neurosurgery in China[13].Moreover, we found that decompressive craniectomy (DC) in refractory ICP was performed in Chinese centers more often than that in European centers[15]. This procedure is recommended (level II A evidence) in the Brain Trauma Foundation guidelines[16].

We had performed craniectomy as the primary procedure for TBI patients with clear operative indications to prevent expected postsurgical increases in ICP and hypothesized that there are subpopulations of patients who need surgical intervention will benefit from primary DC. However, the precise characteristics of these subpopulations are not, as yet, clearly defined. The primary purpose of this study is to assess the impact of DC on subgroups of STBI by comparing the 6-month mortality between primary DC and standard craniotomy. And also, to determine independent predictors and develop a multivariate logistic regression equation to predict who should undergo and benefit from the primary DC.

\section{Methods}

\section{Study Description}

This is a retrospective cohort study which includes 245 adult patients (age of 18 to 65) of severe TBI (Glasgow Coma Scale 6 to 8) with mass lesions admitted to a medical center in China (Tangdu Hospital) from June 2015 through May 2019. This study was approved by the ethics committee of our hospital (No. TDLL2015105). Given the study design of a retrospective chart review, patient consent was not required. All clinical data, including medical records, brain imaging examinations, operation reports, intensive care nursing records, and outpatient visit reports were retrospectively reviewed.

\section{Operative Indications}


All sTBI patients with a score of 6 to 8 on the GCS undergoing craniotomy or primary DC following head injury during this period were included in the study. The key indications for operation were as follows:

Frontal or temporal cerebral contusions greater than $20 \mathrm{~cm}^{3}$ in volume with midline shift of at least $5 \mathrm{~mm}$ and/or cisternal compression of CT scan. Any lesion greater than $50 \mathrm{~cm}^{3}$ in volume[17]. Some patients received simple craniotomy with evacuation of hematoma. Whereas others received primary DC in whom were left with the "bone-off" after mass lesion evacuation. The decision for primary DC was based on neurosurgeon discretion, some patients were decided to DC before in the operating room, some of them the bone flap was not repositioned due to the presence of major intraoperative brain swelling during operation, there was no fixed protocol.

\section{Surgical Technique}

For analysis, patients were divided in two groups: craniotomy group and DC group. Selection of initial surgical procedure was nonrandomized, and the choice of surgical approach was at the discretion of the attending neurosurgeon. All surgeries were conducted by a well-trained surgical team. Briefly, patients in craniotomy group underwent either large unilateral frontotemporoparietal craniectomy (hemicraniectomy), which was recommended for patients with unilateral hemispheric swelling, or bifrontal craniectomy, which was recommended for patients with diffuse brain swelling that affected both hemispheres according to the position of hematomas on imaging studies. The exact type of craniectomy was left to the discretion of the surgeons. And a frontotemporoparietal bone flap was created in DC group. In both groups, the dura was coagulated and widely opened. A relaxation suture duraplasty was performed by synthetic material or by a periosteal patch. An ICP microsensor was placed in the parenchyma of the ipsilateral frontal lobe of each patient.

\section{Treatment}

All patients were managed in the intensive unit with standard medical treatment and critical care according to the guideline for the Management of Severe Traumatic Brain Injury 4th Edition by Brian Trauma Foundation[16]. CT scans, biochemical examinations, routine blood tests, and routine coagulation studies were performed immediately when the patients were admitted to the emergency department. The medical history and results of the neurologic physical examination were recorded immediately after admission. Vital signs were monitored. Treatment protocols target cerebral perfusion pressure (CPP) >60 mm Hg冈ICP <20 mm Hg , PaO2 80-120 mm Hg, PaCO2 35-40 mmHg, hemoglobin $>90 \mathrm{~g} / \mathrm{L}$, temperature $<37.5 \mathrm{C}$, and sodium $>140 \mathrm{mmol} / \mathrm{L}$. Patients were appropriate sedation and analgesia with a continuous intravenous infusion of midazolam and fentanyl. If needed, hypertonic saline (3\%) or mannitol was used.

\section{Data Collection}

The patient's basic information (age, gender, the mode of injury, the initial GCS score, etc) was recorded and retrospectively from our hospital's computerized electronic database. GCS, length of stay, time to various procedures, and all other vital signs were collected daily from admission until the removal of ICP 
monitor or death. ICP mean values were recorded each hour. The patients were followed up by telephone by three trained assessors to record their prognostic information at 6 months after surgery. The outcome of patients at 6 months after injury was categorized according to the GOSE that ranges from 1 to 8 , with lower scores indicating a poorer functional outcome[18]. A score of 5-8 was considered a favorable outcome (moderate disability or less) and a score of 1-4 was considered unfavorable (severe disability or death).

The midline shift at the Foramen of Monro on the CT scan was defined as the absolute distance $(\mathrm{cm})$ that the septum pellucidum of the brain was displaced away from the midline, which was determined as an average by calculating the distance between both inner tables inside the skull[19].For convenience of data analysis, the presence of a fixed pupil was defined as unresponsive $(<1 \mathrm{~mm})$ to a light stimulus. The pupillary light reflex was divided into three categories including one pupil fixed, both pupils fixed and both pupils reactive[20].

Cerebrospinal fluid cisterns around the midbrain are divided into three limbs. Basal cisterns were also dived into three categories: open (all limbs open), partially closed (one or two limbs obliterated), and completely closed (all limbs obliterated)[17].

\section{Statistical Analysis}

Primary analysis was to compare 6-month mortality and functional outcomes between groups. The GOSE score was dichotomized as poor outcome (GOSE 1-4) and favorable outcome (GOSE 5-8). The data were analyzed using version 17.0 of SPSS (SPSS, Chicago, IL, USA) software. Variables were classified as continuous or categorical. Independent Student's t-tests were used for continuous variables between the two groups. Fisher's exact test or Pearson's $\chi 2$ test was used for comparison of categorical variables among groups. Multiple logistic regression models were used for correlation between two continuous or categorical variables and the independent risk factors for DC. Finally, logistic regression analysis was then used to establish a prognostic model and a ROC curve was then drawn based on the prognostic scoring model [21]. A two-tailed $P$ value $<0.05$ was considered statistically significant.

\section{Results}

\section{Patients}

From June 2015 to May 2019, a total of 947 adult consecutive patients with TBI admitted to our hospital were reviewed retrospectively. Of these, 245 patients with a score of 6 to 8 o the GCS who underwent craniotomy or DC were retrospectively evaluated in this study. Patients were divided into two groups: craniotomy group and DC group (Table 1). There were 152 patients in the DC group and 93 patients in the craniotomy group. The mean age was $48.29 \pm 14.59$ years in the DC group, while $47.98 \pm 14.72$ years in the craniotomy group, there was no significant difference $(\mathrm{p}=0.874, \mathrm{t}=0.159)$. Male patients were significantly predominant at $76.97 \%(117 / 152)$ and $80.64 \%$ (75/93) in the DC group and craniotomy group, respectively, with no difference between two groups $(p=0.527, \chi 2=0.498)$. The mean systolic blood 
pressure was $136.98 \pm 26.84 \mathrm{mmHg}$ and $133.67 \pm 20.33 \mathrm{mmHg}$ in the DC group and craniotomy group, respectively, without significant difference between two groups $(p=0.276, t=1.09)$. The proportion of patients with systolic blood pressure higher than $140 \mathrm{mmHg}$ was similar between the two groups $\left(p=0.893, \chi^{2}=0.034\right), 38.81 \%(59 / 152)$ in the DC group and $37.63 \%(35 / 93)$ in the craniotomy group, respectively. Patient in the DC group had a lower mean GCS than that in the craniotomy group $(p=0.01$, $\mathrm{t}=2.603$ ), the mean GCS was $6.75 \pm 0.8$ in the $\mathrm{DC}$ group and $7.04 \pm 0.88$ in the craniotomy group, respectively. Although, the median GCS was same and both were 7 in two groups, there were much more patients with GCS 6 in the DC group than that in the craniotomy group $(p=0.007, \chi 2=9.78)$. Both the mean midline shift and the proportion of midline shift more than $0.5 \mathrm{~cm}$ were similar in two groups, the mean midline shift was $0.43 \pm 0.33 \mathrm{~cm}$ in the DC group in which $43.42 \%$ was more than $0.5 \mathrm{~cm}$, while the mean midline shift in the craniotomy group was $0.38 \pm 0.28 \mathrm{~cm}$ in which $39.78 \%$ was more than $0.5 \mathrm{~cm}$. The mean time from injury to surgery was similar in the two groups, that was $15.06 \pm 15.27$ hours and $16.32 \pm 14.86$ hours for the DC group and the craniotomy group, respectively. The pre-operation pupillary light reflex was significantly different in the two groups $\left(p=0.000, \chi^{2}=71.67\right)$, there were much more patients with one or both pupillary fixed in the DC group than that in the craniotomy group. Similarly, there were much more patients with closed basal cisterns in the DC group than that in the craniotomy group $\left(p=0.000, \chi^{2}=54.54\right)$. For pre-operation CT imaging, there were much more patients with diffuse injury in the DC group than that in the craniotomy group $\left(p=0.025, \chi^{2}=5.53\right)$. Patients and their clinical characteristics are summarized in Table 1.

\section{Outcomes}

The overall 6-month mortality in the entire cohort was $11.43 \%(28 / 245)$. The 6 -month mortality in the DC group was $15.13 \%(23 / 152)$ which was higher than that in the craniotomy group $(5.37 \%, 5 / 93)(p=0.022$, $\left.\chi^{2}=5.42\right)$. However, in the surviving patients, the favorable GOSE rate was similar in two groups $(p=0.061)$, the favorable GOSE rate in the DC group was $78.29 \%$ and in the craniotomy group was $87.5 \%$. The 6month mortality and GOSE are summarized in Table 2.

To test the hypothesis that there are subpopulations of patients who need surgical intervention will benefit from primary DC. Since there were significantly varies in GCS, pupillary light reflex, basal cisterns, and CT imaging in the two groups which might have different associations to the patient's outcomes, subgroup analysis was also performed. Patients were further divided into several subgroups according to GCS, pupillary light reflex, basal cisterns, and CT imaging, respectively. The mortality and the favorable GOSE rate were compared in subgroups.

Although there were more patients with GCS 6 in the DC group than in the craniotomy group, the mortality and the favorable GOSE rate in DC group was similar with the craniotomy group (Table 3). That were similar situations for patients with GCS 7 or GCS 8, the mortality was higher, and the favorable GOSE rate was lower in the DC group than the craniotomy group without significantly difference. 
For patients with at least one pupillary light reflex present, the mortality was same in the DC group and the craniotomy group, and the favorable GOSE rate was similar in the two groups (Table 4). However, for patients with both pupillary light reflex absent, the mortality was lower in the DC group and the favorable GOSE rate was significantly higher in the DC group than the craniotomy group which indicated that this subgroup of patients might be benefit from the DC operation.

There were significantly less patients with basal cisterns open in the DC group than the craniotomy group, while significantly more patients with closed basal cisterns in the DC group $\left(p=0.000, \chi^{2}=54.54\right)$ which consistent with the fact that more severe TBI patients were more likely to choose DC (Table 5). For patients with basal cisterns open, the mortality was significantly higher in the DC group than in the craniotomy group, and the favorable GOSE rate was significantly lower in the DC group. However, for patients with closed basal cisterns, the mortality was lower in the DC group and the favorable GOSE rate was significantly higher in the DC group than the craniotomy group which indicated that this subgroup of patients might be benefit from the DC operation. For patients with partial closed basal cisterns, the mortality and the favorable GOSE rate was similar in the two groups.

There were significantly more patients with diffuse injury showed by CT imaging in the DC group than the craniotomy group $\left(p=0.025, \chi^{2}=5.53\right)$. For patients with diffuse injury, the mortality and the favorable GOSE rate was similar in the two groups (Table 6). The mortality in patients with mass lesion in the DC group tended to increase, but there was not significantly difference. However, the favorable GOSE rate was significantly lower in the DC group than the craniotomy group $\left(p=0.011, \chi^{2}=7.11\right)$ which indicated that this subgroup of patients might not be benefit from the DC operation.

Multivariate logistic regression was then performed on the significant variables extracted from the previous step to determine the independent associations of each variable with the choice of DC (Table 7). Thus, the final model contained two variables: pupillary light reflex and basal cisterns. These results showed pupillary light reflex $(\mathrm{OR}=7.51, \mathrm{Cl}=2.34-24.07 ; p<0.001)$, and basal cisterns $(\mathrm{OR}=4.22, \mathrm{Cl}$ $=1.42-12.59 ; p=0.01)$ were independent of associations to the choice of DC.

At the same time, a predicted probability $(P)$ of choosing DC was estimated by the multiple logistic regression model: $\operatorname{Ln}(P / 1-P)=)=-2334+1.44 \times($ basal cisterns $)+2.016 \times$ (pupil reaction). The predictors of the model were selected by stepwise procedure. Using ROC curve analysis based on the prognostic model scoring, a cut off point for prediction of choosing DC of $p$ was defined as a value $=3.19$. The sensitivity of the equation was $81.6 \%$, and specificity $84.9 \%$ in predicting the choice of DC for operation after TBI (AUC=0.811, 95\% Cl=0.75-0.87, Figure 1).

\section{Discussion}

In this study, we evaluated the impact of primary DC and craniotomy on STBI with mass lesions. As expected (given the greater severity), patients undergoing primary DC had higher 6-month mortality than the remainder of the cohort. However, in the surviving patients, the favorable GOSE rate was similar in 
primary DC patients and craniotomy patients indicated that primary DC might improve outcomes of subgroup patients of sTBI. We further identified that patients either with both pupillary light reflex absent or with closed basal cisterns, the primary DC decreased the mortality and increased the favorable GOSE proportion. Furthermore, we developed a multivariate logistic regression equation for decision making and to determine whether the primary DC should be done or not for STBI with mass lesion.

TBI is one of the leading causes of death and disability worldwide, with a global annual incidence of more than 50 million cases[22]. With a population of 1.4 billion, the number of patients with TBI in China exceeds that of most other countries, causing an enormous burden to society and families[1]. Among patients who are hospitalized with severe $\mathrm{TBI}, 60 \%$ either die or survive with severe disability[18]. In China, the proportion of patients with severe TBI is around $20 \%$,and the mortality of sTBI is also around $20 \%[2$, 13].

After severe TBI, medical and surgical therapies are performed to minimize secondary brain injury[18]. The treatment goal of severe TBI is to reduce and prevent intracranial hypertension (ICH), a pathologic increase in ICP can lead to brain ischemia by reducing CPP and lead to neurologic deficit and fatal brain herniation syndromes. To achieve this goal, several medical and surgical therapies can be performed, including head-of-bed elevation, pharmacologic sedation and analgesic, improved blood flow, external ventricular drain, surgical evacuation of hemorrhagic lesions, and DC[23]. Despite recommendations by the Brain Trauma Foundation supporting the use of these therapies for sTBI, there is no level I evidence demonstrating improvement in patient outcomes through definitive, surgical management of TBI [17, 24]. DC has been performed for the purpose of relieving elevated intracranial pressure with outcome improvement in specific TBI patients[25].DC holds the promise of reduced mortality, but long term neurological outcomes and indications remain controversial[10].

Recently, in The Lancet Neurology, the results of the Chinese sister study-the CENTER-TBI China registry study including 13138 patients from 52 centers showed that surgical interventions, including insertion of the intracranial pressure device, decompression, external ventricular drainage, and hematoma removal show therapeutic benefits in patients with signs of brain herniation[2]. Moreover, this finding probably reflects the high proportion of patients with severe injury, but from a clinical perspective preemptively treating impending brain herniation is preferable, rather than waiting for the condition's full development[2]. The effectiveness of these surgical interventions in this cohort is of particular relevance given the lack of benefit reported in the overall TBI population in previous clinical trials[26-28]. The identification of subgroups who are most likely to benefit from these interventions should be a priority[2].

In China, the indications for unilateral or bilateral large DC include progressive neurological deterioration, intracerebral hematoma, contusion or edema with midline shift more than $5 \mathrm{~mm}$ and cisternal compression on CT, and intracranial pressure higher than $30 \mathrm{~mm} \mathrm{Hg}$ for longer than $30 \mathrm{~min}$ [13]. However, these indication variables alone do not define the patient who should undergo DC intervention. Although, intracranial pressure monitoring is widely used to evaluate the intracranial status of patients with TBI. And, in the past two decades, the addition of ICP monitoring has markedly improved decision making and 
management of patients with severe TBI[13]. But, most patients admitted to the ICU with severe TBI in China did not receive an ICP monitoring device, thus implying that in many centers treatment decisions are made on the basis of clinical and radiological findings[2]. Instead, in China, CT scans are done for patients with TBI in all hospitals with departments of neurosurgery[1]. Primary DC is most often performed for clinical and radiographic evidence of herniation, rather than for refractory ICP elevation[29].Moreover, we found that DC in TBI was performed in Chinese centers more often than that in European centers[2, 15].

Most sTBI patients with intracranial hypertension attribute to mass lesions, such as contusion and acute subdural hematoma (ASDH)[17]. Severe primary injury and worsening condition necessitate emergent surgical intervention. The available type of operation is either craniotomy or DC. Generally, there are three distinct scenarios in which DC is performed for TBI: firstly, as a "primary" procedure following evacuation of a mass lesion; secondly, as emergent treatment for neurological deterioration attributable to worsening mass effect in patients who previously were not considered to have an indication for ICP monitoring; and thirdly, as treatment for raised ICP that is refractory to medical therapy[29].

In this study, in contrast to DECRA and RESCUE-ICP $[18,27]$, we performed DC as primary procedure following evacuation of a mass lesion. Our data supported the hypothesis that some specific TBI patients would benefit from primary DC. Our results in line with some studies which suggesting primary DC proposed that early aggressive intervention could mitigate the secondary damages of increased ICP[30]. In Europe, a retrospective study of 729 patients revealed that one-third of patients with STBI who received emergency surgery still needed DC even after hematoma evacuation[31]. In a retrospective cohort comparison study of 91 patients who had an operation for an ASDH, 56\% received a primary DC, while the rest a craniotomy[32]. This study supports the hypothesis that a primary DC (i.e. bone flap left out after ASDH evacuation) may lead to better outcomes compared to a craniotomy (i.e. bone flap is replaced) due to better control of brain swelling and intracranial hypertension in the post-operative period. This hypothesis is also supported by a two-center non-experimental comparative effectiveness research (CER), which found that post-operative ICP was better controlled and patient outcomes were better in the center with greater utilization of primary $\mathrm{DC}[33,34]$. Given the potential benefit from primary DC on TBI, there are several prospective randomized trials are going on. The primary DC for patients with ASDH is being systematically evaluated in the context of the RESCUE-ASDH trial in UK[33]. And, the effect of primary DC in STBI with mass lesions is being evaluated in a prospective, randomized, assessor-blind, single center clinical trial named prospective, randomized evaluation of therapeutic decompressive craniectomy in STBI with mass lesions (PRECIS) in China[35].

When comparing DC with craniotomy patients, it shows DC patients have a lower mean preoperative GCS $(p=0.01)$; more patients with GCS of $6(\mathrm{p}=0.007)$;more unresponsive pupillary light reflex $(p<0.001)$; more closed basal cisterns $(p<0.001)$; and more patients with diffuse injury $(\mathrm{p}=0.025)$. These results seem to support the notion that the perioperative neurological status in decompressive craniectomy patients is more severe than in craniotomy. To explore the factors involved in decision marking for DC, in addition to the single factor analysis and multi-factorial prognostic analysis, in our study, we further 
combined an ROC curve and the multivariate logistic regression equation to evaluate the predictive accuracy of two variables in choosing of DC. In line with previous reports demonstrated that the effacement of the basal cisterns and the absent of pupil reaction are strongly associated with poor outcomes and that calling for DC[36]. These results disclosed two variables, which had previously been shown to be related to survival, as having strong accuracy in prognostic judgment with sensitivity $81.6 \%$ and specificity $84.9 \%$ in predicting of DC. Since these two variables in the scoring model are clinically simple to attain in the acute stage after TBI, we consider therefore, that the derived equation is clinically useful to make decision on whether $\mathrm{DC}$ or not in daily practice.

There are limitations of the present study must be considered because of the nature of the retrospective, nonrandomized design of this study. It is a retrospective analysis of a 5-year cohort of sTBI patients with mass lesion requiring primary DC or craniotomy. The decision of operation procedure was nonrandom that may be affected by experience of surgeons and patients' condition. The perioperative neurological status in patients with DC is more severe than in craniotomy. This issue needs to be addressed in prospective studies. Another limitation of this study is lacking preoperative ICP monitoring. Although, all the patients in this study need operation, without preoperative ICP, we could not analyze the ICP factors in decision making. Additional limitation of the present study includes the heterogeneity of the patient population. Despite all patients in present study with mass lesions, the different types of TBI such as epidural hematoma, subdural hematoma and contusion hemorrhage will result in different outcomes and might vary in operation style. In future, the subgroup analysis of an enriched population of different types of TBI patients is needed to clarify the issue.

\section{Conclusion}

Based on our findings, we confirm that the primary DC may benefit subgroups of sTBI with mass lesions. We conclude that two factors: unresponsive pre-op pupil reaction, and closed basal cistern are associated with primary DC chosen in patients with STBI with mass lesions. These factors can be used as independent predictors in assessing the decision making on DC in TBI patients. The two variables derived multivariate logistic equation is also clinically useful for predicting utilization of DC or not in daily practice.

\section{Declarations}

\section{Acknowledgements}

Not applicable.

\section{Authors' contributions}

CY and JRZ designed, analyzed, wrote, processed, and reviewed the manuscript. GZ, HG, FG, BW, WXC, YWS and YD helped in concept, data collection, materials, and literature search. ZHL and LW helped in 
resources, materials, data collection and literature search. LTM, YQ and SNG designed, supervised, analyzed, and reviewed the manuscript. All authors read and approved the final manuscript.

\section{Funding}

This work was supported by the National Natural Science Foundation of China (grant No. 81701304), the China Postdoctoral Science Foundation (grant No.2017M623432)

\section{Availability of data and materials}

Data and materials in the report is collected from our own cases. All the data supporting the conclusions of this article is included in the present article.

\section{Ethics approval and consent to participate}

Although the study was in the category of noninterventional clinical research with its retrospective nature, we did apply for ethics committee approval. The ethics committee approved the study (No. TDLL2015105) and formal consent in addition to what the patients had given prior to hospitalization was waived.

\section{Consent for publication}

Not applicable.

\section{Competing interests}

The authors declare that they have no competing interests.

\section{Author details}

1Department of Neurosurgery, Tangdu Hospital, The Fourth Military Medical University, Xi'an, Shaanxi 710038, China. 2 Postdoctoral Research Station of Neurosurgery, General Hospital of Central Theater Command of Chinese People's Liberation Army, Wuhan, Hubei 430064, China. 3 The Helmholtz SinoGerman Laboratory for Cancer Research, Department of Pathology, Tangdu Hospital, The Fourth Military Medical University, Xi'an, Shaanxi 710038, China

\section{Abbreviations}

ASDH: acute subdural hematoma; CPP: cerebral perfusion pressure; CT: Computed tomography; DC: Decompressive craniectomy; GCS: Glasgow Coma Scale; GOSE: Extended Glasgow Outcome Scale; ICH: intracranial hypertension; ICP: Intracranial pressure; ICU: Intensive Care Unit; ROC: Receiver operating characteristic curves; TBI: Traumatic brain injury. 


\section{References}

1. Jiang JY, Gao GY, Feng JF, Mao Q, Chen LG, Yang XF, et al. Traumatic brain injury in China. LANCET NEUROL 2019 2019-03-01;18(3):286-95.

2. Gao G, Wu X, Feng J, Hui J, Mao Q, Lecky F, et al. Clinical characteristics and outcomes in patients with traumatic brain injury in China: a prospective, multicentre, longitudinal, observational study. LANCET NEUROL 2020 2020-08-01;19(8):670-7.

3. Hawley C, Sakr M, Scapinello S, Salvo J, Wrenn P. Traumatic brain injuries in older adults-6 years of data for one UK trauma centre: retrospective analysis of prospectively collected data. EMERG MED J 2017 2017-08-01;34(8):509-16.

4. Singounas EG. Severe head injury in a paediatric population. J NEUROSURG SCI 1992 1992-1001;36(4):201-6.

5. Bullock R, Golek J, Blake G. Traumatic intracerebral hematoma-which patients should undergo surgical evacuation? CT scan features and ICP monitoring as a basis for decision making. Surg Neurol 1989 1989-09-01;32(3):181-7.

6. Soloniuk D, Pitts LH, Lovely M, Bartkowski H. Traumatic intracerebral hematomas: timing of appearance and indications for operative removal. J Trauma 1986 1986-09-01;26(9):787-94.

7. Kolias AG, Belli A, Li LM, Timofeev I, Corteen EA, Santarius T, et al. Primary decompressive craniectomy for acute subdural haematomas: results of an international survey. Acta Neurochir (Wien) 2012 2012-09-01;154(9):1563-5.

8. Timofeev I, Santarius T, Kolias AG, Hutchinson PJ. Decompressive craniectomy - operative technique and perioperative care. Adv Tech Stand Neurosurg 2012 2012-01-20;38:115 - 36.

9. Kolias AG, Kirkpatrick PJ, Hutchinson PJ. Decompressive craniectomy: past, present and future. NAT REV NEUROL 2013 2013-07-01;9(7):405-15.

10. Sahuquillo J, Dennis JA. Decompressive craniectomy for the treatment of high intracranial pressure in closed traumatic brain injury. Cochrane Database Syst Rev 2019 2019-12-31;12:D3983.

11. Gouello G, Hamel O, Asehnoune K, Bord E, Robert R, Buffenoir K. Study of the long-term results of decompressive craniectomy after severe traumatic brain injury based on a series of 60 consecutive cases. ScientificWorldJournal 2014 2014-01-20;2014:207585.

12. Honeybul S, Ho KM, Lind CR. What can be learned from the DECRA study. WORLD NEUROSURG 2013 2013-01-01;79(1):159-61.

13. Jiang JY. Head trauma in China. INJURY 2013 2013-11-01;44(11):1453-7.

14. Maegele M. Global traumatic brain injury research enters a new era. LANCET NEUROL 2020 2020-0801;19(8):637-9.

15. Feng J, van Veen E, Yang C, Huijben JA, Lingsma HF, Gao G, et al. Comparison of Care System and Treatment Approaches for Patients with Traumatic Brain Injury in China versus Europe: A CENTERTBI Survey Study. J Neurotrauma 2020 2020-08-15;37(16):1806-17. 
16. Carney N, Totten AM, O'Reilly C, Ullman JS, Hawryluk GW, Bell MJ, et al. Guidelines for the Management of Severe Traumatic Brain Injury, Fourth Edition. NEUROSURGERY 2017 2017-01$01 ; 80(1): 6-15$.

17. Bullock MR, Chesnut R, Ghajar J, Gordon D, Hartl R, Newell DW, et al. Surgical management of traumatic parenchymal lesions. NEUROSURGERY 2006 2006-03-01;58(3 Suppl):S25-46.

18. Cooper DJ, Rosenfeld JV, Murray L, Arabi YM, Davies AR, D'Urso P, et al. Decompressive craniectomy in diffuse traumatic brain injury. N Engl J Med. 2011;2011-04-21(16):1493-502. 364(.

19. Lobato RD, Rivas JJ, Gomez PA, Castaneda M, Canizal JM, Sarabia R, et al. Head-injured patients who talk and deteriorate into coma. Analysis of 211 cases studied with computerized tomography. J NEUROSURG 1991 1991-08-01;75(2):256-61.

20. Kuo JR, Lo CJ, Lu CL, Chio CC, Wang CC, Lin KC. Prognostic predictors of outcome in an operative series in traumatic brain injury patients. J FORMOS MED ASSOC 2011 2011-04-01;110(4):258 - 64.

21. Zweig MH, Campbell G. Receiver-operating characteristic (ROC) plots: a fundamental evaluation tool in clinical medicine. CLIN CHEM 1993 1993-04-01;39(4):561-77.

22. Maas A, Menon DK, Adelson PD, Andelic N, Bell MJ, Belli A, et al. Traumatic brain injury: integrated approaches to improve prevention, clinical care, and research. LANCET NEUROL 2017 2017-1201;16(12):987-1048.

23. Stocchetti N, Maas Al. Traumatic intracranial hypertension. N Engl J Med 2014 2014-0529;370(22):2121-30.

24. Millar K, Eljamel S. Does Size and Site Matter in Therapeutic Decompressive Craniectomy? A Laboratory-Based Experimental Study. WORLD NEUROSURG 2016 2016-11-01;95:441-6.

25. Bor-Seng-Shu E, Figueiredo EG, Amorim RL, Teixeira MJ, Valbuza JS, de Oliveira MM, et al. Decompressive craniectomy: a meta-analysis of influences on intracranial pressure and cerebral perfusion pressure in the treatment of traumatic brain injury. J NEUROSURG 2012 2012-0901;117(3):589-96.

26. Bales JW, Bonow RH, Buckley RT, Barber J, Temkin N, Chesnut RM. Primary External Ventricular Drainage Catheter Versus Intraparenchymal ICP Monitoring: Outcome Analysis. NEUROCRIT CARE 2019 2019-08-01;31(1):11-21.

27. Hutchinson PJ, Kolias AG, Timofeev IS, Corteen EA, Czosnyka M, Timothy J, et al. Trial of Decompressive Craniectomy for Traumatic Intracranial Hypertension. N Engl J Med 2016 2016-0922;375(12):1119-30.

28. Chesnut RM, Temkin N, Carney N, Dikmen S, Rondina C, Videtta W, et al. A trial of intracranialpressure monitoring in traumatic brain injury. N Engl J Med. 2012;367(26):2471-81. 2012-12-27.

29. Kramer AH, Deis N, Ruddell S, Couillard P, Zygun DA, Doig CJ, et al. Decompressive Craniectomy in Patients with Traumatic Brain Injury: Are the Usual Indications Congruent with Those Evaluated in Clinical Trials? NEUROCRIT CARE 2016 2016-08-01;25(1):10-9.

30. Weiner GM, Lacey MR, Mackenzie L, Shah DP, Frangos SG, Grady MS, et al. Decompressive craniectomy for elevated intracranial pressure and its effect on the cumulative ischemic burden and 
therapeutic intensity levels after severe traumatic brain injury. NEUROSURGERY 2010 2010-0601;66(6):1111-8, 1118-9.

31. Compagnone C, Murray GD, Teasdale GM, Maas Al, Esposito D, Princi P, et al. The management of patients with intradural post-traumatic mass lesions: a multicenter survey of current approaches to surgical management in 729 patients coordinated by the European Brain Injury Consortium. NEUROSURGERY 2005 2005-12-01;57(6):1183-92, 1183-92.

32. Li LM, Kolias AG, Guilfoyle MR, Timofeev I, Corteen EA, Pickard JD, et al. Outcome following evacuation of acute subdural haematomas: a comparison of craniotomy with decompressive craniectomy. Acta Neurochir (Wien) 2012 2012-09-01;154(9):1555-61.

33. Kolias AG, Adams H, Timofeev I, Czosnyka M, Corteen EA, Pickard JD, et al. Decompressive craniectomy following traumatic brain injury: developing the evidence base. $\mathrm{Br} \mathrm{J}$ Neurosurg 2016 2016-01-20;30(2):246-50.

34. Hartings JA, Vidgeon S, Strong AJ, Zacko C, Vagal A, Andaluz N, et al. Surgical management of traumatic brain injury: a comparative-effectiveness study of 2 centers. J NEUROSURG. 2014;2014-0201(2):434-46. 120(.

35. Zhao HX, Liao Y, Xu D, Wang QP, Gan Q, You C, et al. Prospective randomized evaluation of therapeutic decompressive craniectomy in severe traumatic brain injury with mass lesions (PRECIS): study protocol for a controlled trial. BMC NEUROL 2016 2016-01-05;16:1.

36. Maas Al, Hukkelhoven CW, Marshall LF, Steyerberg EW. Prediction of outcome in traumatic brain injury with computed tomographic characteristics: a comparison between the computed tomographic classification and combinations of computed tomographic predictors. NEUROSURGERY 2005 2005-12-01;57(6):1173-82, 1173-82.

\section{Tables}

Table 1. Characteristics of severe traumatic brain injury patients with mass lesions undergoing decompressive craniectomy or craniotomy 


\begin{tabular}{|c|c|c|c|}
\hline Characteristics & $\begin{array}{l}\text { DC } \\
(n=152)\end{array}$ & $\begin{array}{l}\text { craniotomy } \\
(n=93)\end{array}$ & $\mathbf{p}$ \\
\hline Age (years) & $48.29 \pm 14.59$ & $47.98 \pm 14.72$ & 0.874 \\
\hline Male & 117ه76.97\%囚 & $75 \bowtie 80.64 \% \rrbracket$ & 0.527 \\
\hline Systolic blood pressure $(\mathrm{mmHg})$ & $136.98 \pm 26.84$ & $133.67 \pm 20.33$ & 0.276 \\
\hline$>140 \mathrm{mmHg}$ & $59 \llbracket 38.81 \% \rrbracket$ & $35 \bowtie 37.63 \% \rrbracket$ & 0.893 \\
\hline GCS(median) & $6.75 \pm 0.80(7)$ & $7.04 \pm 0.88(7)$ & 0.01 \\
\hline 6 & 72 & 33 & 0.007 \\
\hline 7 & 45 & 21 & \\
\hline 8 & 35 & 39 & \\
\hline midline shift(cm) & $0.43 \pm 0.33$ & $0.38 \pm 0.28$ & 0.22 \\
\hline$>0.5$ & $66(43.42 \%)$ & $37(39.78 \%)$ & 0.596 \\
\hline Time from injury to surgery(hours) & $15.06 \pm 15.27$ & $16.32 \pm 14.86$ & 0.432 \\
\hline Pupillary light reflex & & & 0.000 \\
\hline Both present & $23(5.13 \%)$ & $62(66.66 \%)$ & \\
\hline One absent & $64(42.10 \%)$ & $23(24.73 \%)$ & \\
\hline Bth absent & $65(42.76 \%)$ & $8(8.60 \%)$ & \\
\hline basal cisterns & & & 0.000 \\
\hline Open & $32(21.05 \%)$ & $57(61.29 \%)$ & \\
\hline Partial closed & $41(26.97 \%)$ & $27(29.03 \%)$ & \\
\hline Closed & $79(51.97 \%)$ & $9(12.12 \%)$ & \\
\hline \multicolumn{4}{|l|}{ CT imaging } \\
\hline With diffuse injury & $84(55.26 \%)$ & $37(39.78 \%)$ & 0.025 \\
\hline Mass lesion only & $68(44.74 \%)$ & $56(60.22 \%)$ & \\
\hline
\end{tabular}

Table 2. The 6-month mortality and GOSE 


\begin{tabular}{|llll|}
\hline GOSE & $\begin{array}{l}\text { DC } \\
(\mathbf{n = 1 5 2})\end{array}$ & $\begin{array}{l}\text { craniotomy } \\
(\mathbf{n = 9 3})\end{array}$ & $\mathbf{p}$ \\
\hline 1 (dead) & $23(15.13 \%)$ & $5(5.37 \%)$ & 0.022 \\
\hline 2 & $4(2.63 \%)$ & $2(2.15 \%)$ & \\
\hline 3 & $19(12.5 \%)$ & $4(4.3 \%)$ & \\
\hline 4 & $3(1.97 \%)$ & $3(3.22 \%)$ & \\
\hline 5 & $11(7.23 \%)$ & $8(8.6 \%)$ & $0.061 *$ \\
\hline 6 & $20(13.15 \%)$ & $10(10.75 \%)$ & \\
\hline 7 & $40(26.31 \%)$ & $23(26.88 \%)$ & \\
\hline 8 & $32(21.05 \%)$ & $36(38.7 \%)$ & \\
\hline
\end{tabular}

*In the surviving patients (GOSE of 2 to 8 ), the favorable GOSE rate (GOSE of 5 to 8 ) was similar in two groups $(p=0.061)$, the favorable GOSE rate in the DC group was $78.29 \%$ and in the craniotomy group was $87.5 \%$.

Table 3. The mortality and the favorable GOSE rate were compared in subgroups according to preoperative GCS 


\begin{tabular}{|c|c|c|c|}
\hline & $\begin{array}{l}\text { DC } \\
(n=152)\end{array}$ & $\begin{array}{l}\text { craniotomy } \\
(n=93)\end{array}$ & p \\
\hline GCS & $6.75 \pm 0.80(7)$ & $7.04 \pm 0.88(7)$ & 0.01 \\
\hline 6 & $72(47.37 \%)$ & $33(35.48 \%)$ & \\
\hline Dead & $14(19.44 \%)$ & $5(15.15 \%)$ & 0.786 \\
\hline Favorable & $42(58.33 \% \rrbracket$ & $22(66.67 \% \rrbracket$ & 0.519 \\
\hline 7 & $45(29.61 \%)$ & $21(22.58 \%)$ & \\
\hline Dead & $6(13.33 \%)$ & 0 & 0.166 \\
\hline Favorable & $30(66.67 \% \bigotimes$ & 16(76.19\%区 & 0.569 \\
\hline 8 & $35(23.03 \%)$ & $39(41.94 \%)$ & \\
\hline Dead & $3(8.57 \%)$ & 0 & 0.101 \\
\hline Favorable & $31(88.57 \%)$ & $37(94.87 \%)$ & 0.413 \\
\hline
\end{tabular}

Table 4. The mortality and the favorable GOSE rate were compared in subgroups according to preoperative pupillary light reflex 


\begin{tabular}{|llll|}
\hline & $\begin{array}{l}\text { DC } \\
(\mathbf{n = 1 5 2 )}\end{array}$ & $\begin{array}{l}\text { craniotomy } \\
(\mathbf{n = 9 3 )}\end{array}$ & $\mathbf{p}$ \\
\hline Pupillary light reflex & & & 0.000 \\
\hline Both present & $23(15.13 \%)$ & $62(66.66 \%)$ & \\
\hline Dead & 0 & 0 & \\
\hline Favorable & $23(100 \%)$ & $59(95.16 \%)$ & 0.56 \\
\hline One absent & $64(42.10 \%)$ & $23(24.73 \%)$ & \\
\hline Dead & 0 & 0 & \\
\hline Favorable & $54(84.37 \%)$ & $18(78.26 \%)$ & 0.529 \\
\hline Both absent & $65(42.76 \%)$ & $8(8.60 \%)$ & \\
\hline Dead & $23(35.38 \%)$ & $5(62.5 \%)$ & 0.246 \\
\hline Favorable & $24(36.92 \%)$ & 0 & 0.047 \\
\hline & & & \\
\hline
\end{tabular}

Table 5. The mortality and the favorable GOSE rate were compared in subgroups according to preoperative basal cisterns 


\begin{tabular}{|llll|}
\hline & $\begin{array}{l}\text { DC } \\
(\mathbf{n = 1 5 2 )}\end{array}$ & $\begin{array}{l}\text { craniotomy } \\
(\mathbf{n = 9 3})\end{array}$ & $\mathbf{p}$ \\
\hline Basal cisterns & & & 0.000 \\
\hline Open & $32(21.05 \%)$ & $57(61.29 \%)$ & \\
\hline Dead & $4(12.5 \%)$ & 0 & $\mathbf{0 . 0 1 5}$ \\
\hline Favorable & $23(71.87 \%)$ & $52(91.22 \%)$ & $\mathbf{0 . 0 3 1}$ \\
\hline Partial Closed & $41(26.97 \%)$ & $27(29.03 \%)$ & \\
\hline Dead & $4(9.75 \%)$ & 0 & 0.146 \\
\hline Favorable & $33(80.48 \%)$ & $24(88.88 \%)$ & 0.506 \\
\hline Closed & $79(51.97 \%)$ & $9(9.67 \%)$ & \\
\hline Dead & $15(18.98 \%)$ & $5(55.55 \%)$ & $\mathbf{0 . 0 2 6}$ \\
\hline Favorable & $45(56.96 \%)$ & $1(11.11 \%)$ & $\mathbf{0 . 0 1 2}$ \\
\hline & & & \\
\hline
\end{tabular}

Table 6. The mortality and the favorable GOSE rate were compared in subgroups according to preoperative CT imaging

\begin{tabular}{|llll|}
\hline & $\begin{array}{l}\text { DC } \\
(\mathbf{n = 1 5 2})\end{array}$ & $\begin{array}{l}\text { craniotomy } \\
(\mathbf{n = 9 3})\end{array}$ & $\mathbf{p}$ \\
\hline CT imaging & & & 0.025 \\
\hline With diffuse injury & $84(55.26 \%)$ & $37(39.78 \%)$ & \\
\hline Dead & $16(19.04 \%)$ & $4(10.81 \%)$ & 0.302 \\
\hline Favorable & $52(61.90 \%)$ & $26(70.27 \%)$ & 0.416 \\
\hline Mass lesion only & $68(44.74 \%)$ & $56(60.22 \%)$ & \\
\hline Dead & $7(10.29 \%)$ & $1(1.78 \%)$ & 0.069 \\
\hline Favorable & $49(72.05 \%)$ & $51(91.07 \%)$ & 0.011 \\
\hline & & & \\
\hline
\end{tabular}


Table 7. The final model of multiple logistic regression was constructed using the forward stepwise procedure, the adjusted odds ratio in predicting the choice of decompressive craniectomy

\begin{tabular}{|lllll|}
\hline & $\boldsymbol{\beta}$ & OR & $95 \% \mathbf{C L}$ & $\mathbf{p}$ \\
\hline Pupillary light reflex & 2.016 & 7.51 & $2.34-24.07$ & $\mathrm{p}<0.001$ \\
\hline Basal cisterns & 1.441 & 4.22 & $1.42-12.59$ & $\mathrm{p}=0.01$ \\
Constant & -2.334 & & & \\
\hline & & & & \\
\hline
\end{tabular}

Figures 


\section{ROC Curve}

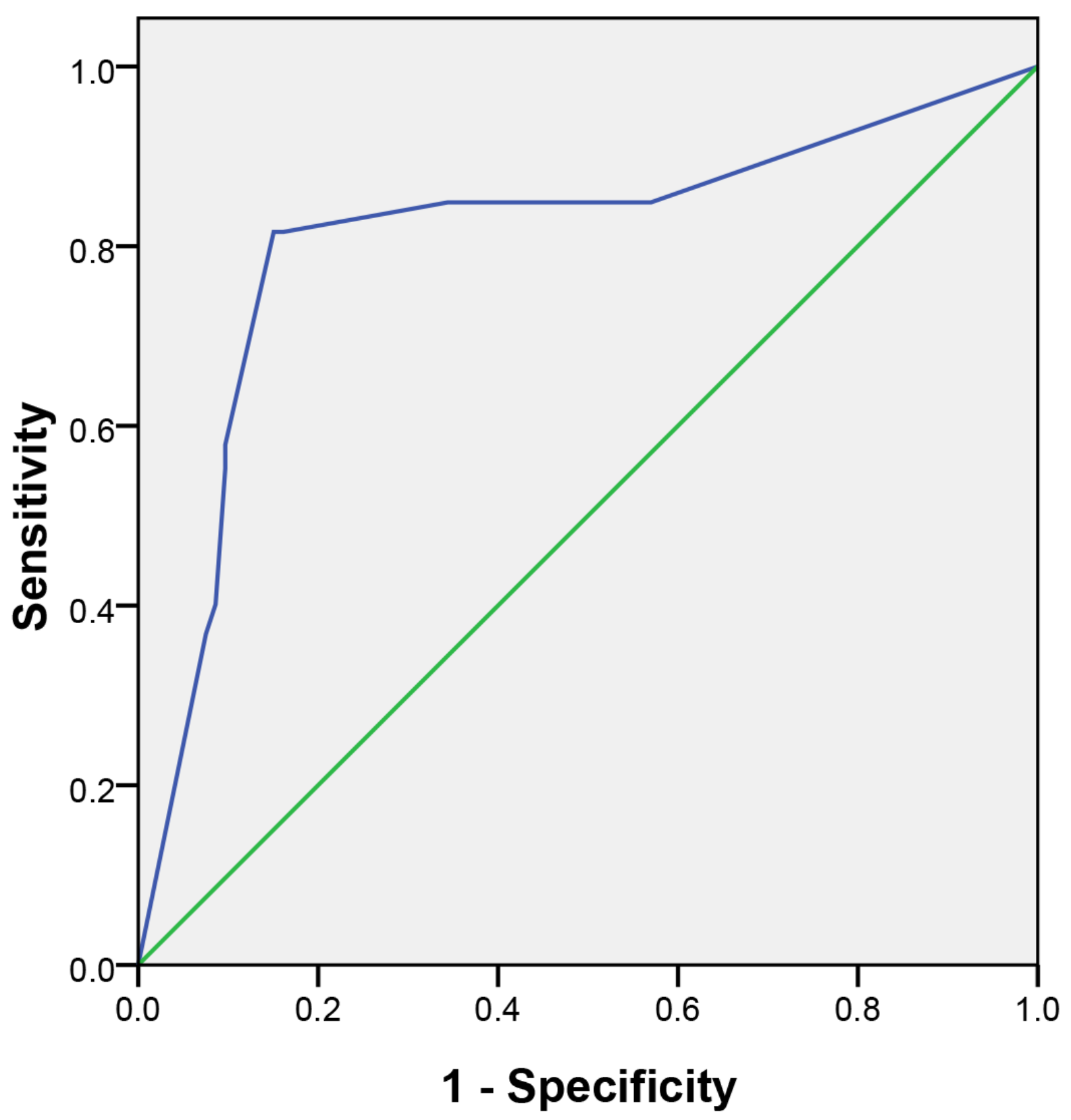

Figure 1

ROC curve. Receiver-operating characteristic curve was drawn based on the prognostic scoring model, logistic $=\operatorname{Ln}(p /(1-p)=-2334+1.44 \times($ basal cisterns $)+2.016 \times($ pupil reaction $\rrbracket$ where, basal cisterns, open $=1$; partial closed $=2$; closed $=3$; pupil reaction, both reactive $=0$, at least one reactive $=1$, both fixed $=2$. 